MMW aufgefrischt

\section{Weniger Balken und Serifen}

Egal ob Damenschuh oder Herrenanzug, was heute als modern empfunden wird, sieht schon bald wieder altmodisch aus. So auch die medizinische Fachzeitschrift. In regelmäßigen Abständen schicken wir uns daher an, das Design Ihrer MMW ein wenig aufzufrischen, wohl wissend, dass dies manchen treuen Stammleser zunächst irritieren mag.

Vor diesem Hintergrund bitten

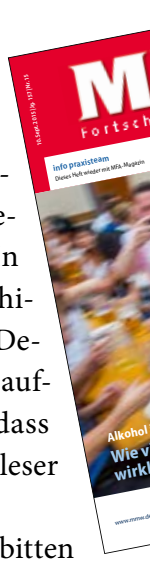
wir um Verständnis, dass wir dennoch etwas Moderne durchsetzen, und beispielsweise den jahrzehntealten Titelschriftzug der MMW mit seinen antiquierten Serifen über Bord spülen. Die zweite Neuerung betrifft die Heftstruktur: Wir trennen künftig noch deutlicher zwischen

- dem aktuellen Medizinteil in Rot,

- dem Praxis-/Wirtschaftsteil in Grün, und

- dem (von wissenschaftlichen Autoren geschriebenen) Fortbildungsteil in Blau.
Dr. med. Dirk Einecke Chefredakteur dirk.einecke@springer.com

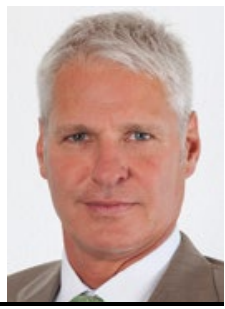

Adipositasrisiko

XL-Portionen machen Appetit auf mehr

Riesenschnitzel, eimerweise Popcorn und Limonade in Literbechern verleiten $\mathrm{zu}$ übermäßiger Kalorienaufnahme. Diese Alltagserfahrung wird durch eine aktuelle Cochrane-Analyse untermauert.

Ausgewertet wurden ausschließlich randomisierte kontrollierte Studien, in denen der Einfluss von unterschiedlichen Größen bzw. Formen von Lebens- oder Genussmitteln auf die Auswahl und/oder den Konsum getestet worden war. Der Effekt, den das Angebot größerer Portionen/Packungen auf das Essverhalten hatte, war gering bis mäßig; die standardisierten mittleren Differenzen (SMD) zwischen den Vergleichsgruppen betrugen 0,21 bei Kindern und 0,46 bei Erwachsenen. Würde ein solcher Unterschied langfristig erhalten bleiben - was in den Studien nicht untersucht wurde - und sich bei der gesamten Ernährung niederschlagen, könnten britische Kinder und Erwachsene auf diese Weise täglich 144-228 kcal einsparen, so die Autoren.

- Cochrane Database of Systematic Reviews 2015, Issue 9. Art. No.: CD011045. DOI: 10.1002/14651858.CD011045.pub2

Leistung objektiv zu messen. Dokumentiert wurde auch die Fähigkeit der Männer, eine Erektion zustande zu bringen hier allerdings verließ man sich auf die Selbstauskünfte.

Insgesamt lag die ED-Quote der 692 Männer bei knapp 54\%. Die Autoren rechneten aus, dass jede Erhöhung der mäßig bis stark belastenden körperlichen Aktivität um täglich eine halbe Stunde das Chancenverhältnis für ED um etwa $40 \%$ vermindert (Odds Ratio [OR] 0,57).

- J Sex Med 2015, online 31. August; doi: 10.1111/jsm.12977
KONGRESS-NEWS AUF SPRINGERMEDIZIN.DE

Highlights vom europäischen Herzkongress lesen Sie in diesem Heft ab S. 8. Ergänzend dazu das Kongressdossier online unter

$\rightarrow$ www.springermedizin.de/esc-2015

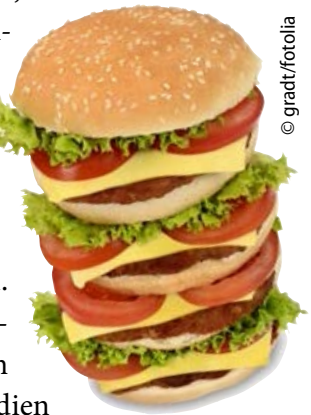

Männer, die mehr Sport treiben, haben Paul Loprinzi und Meghan Edwards von der University of Mississippi haben sich für ihre Querschnittsstudie die Daten des US-National Health and Nutrition Examination Survey (NHANES) vorgenommen. Sie destillierten daraus eine repräsentative Stichprobe von 692 Männern im Alter zwischen 50 und 85 Jahren, die sieben Tage lang mit einem Beschleunigungssensor ausgerüstet worden waren. Dadurch war es möglich, ihre sportliche 